\title{
SPECTRAL STABILITY OF SHIFTED STATES ON STAR GRAPHS
}

\author{
ADILBEK KAIRZHAN AND DMITRY E. PELINOVSKY
}

\begin{abstract}
We consider the nonlinear Schrödinger (NLS) equation with the subcritical power nonlinearity on a star graph consisting of $N$ edges and a single vertex under generalized Kirchhoff boundary conditions. The stationary NLS equation may admit a family of solitary waves parameterized by a translational parameter, which we call the shifted states. The two main examples include (i) the star graph with even $N$ under the classical Kirchhoff boundary conditions and (ii) the star graph with one incoming edge and $N-1$ outgoing edges under a single constraint on coefficients of the generalized Kirchhoff boundary conditions. We obtain the general counting results on the Morse index of the shifted states and apply them to the two examples. In the case of (i), we prove that the shifted states with even $N \geq 4$ are saddle points of the action functional which are spectrally unstable under the NLS flow. In the case of (ii), we prove that the shifted states with the monotone profiles in the $N-1$ outgoing edges are spectrally stable, whereas the shifted states with nonmonotone profiles in the $N-1$ outgoing edges are spectrally unstable, the two families intersect at the half-soliton states which are spectrally stable but nonlinearly unstable under the NLS flow. Since the NLS equation on a star graph with shifted states can be reduced to the homogeneous NLS equation on an infinite line, the spectral instability of shifted states is due to the perturbations breaking this reduction. We give a simple argument suggesting that the spectrally stable shifted states are unstable under the NLS flow due to the perturbations breaking the reduction to the NLS equation on an infinite line.
\end{abstract}

\section{INTRODUCTION}

It is well-known that multi-dimensional models can be reduced approximately to the PDEs on metric graphs in many realistic physical experiments involving wave propagation in narrow waveguides [4, 15, 16, 19]. When a multi-dimensional narrow waveguide is replaced by a graph consisting of edges and vertices, a metric space is introduced for the wave functions on edges and is equipped with appropriate boundary conditions at vertices. Kirchhoff boundary conditions appear naturally in many applications and require the wave functions to be continuous across the vertex points and the sum of the first derivatives of the wave functions to be zero, see, e.g., books [5, 10].

It is relatively less known that the Kirchhoff boundary conditions are not the only possible boundary conditions arising when the narrow waveguides shrinks to a metric graph. By working with different values of the thickness parameters vanishing in the limit at the same rate, it was shown in [23] (see also [9, 11, 12, 18, 20]) that generalized Kirchhoff boundary conditions can also arise in the limit. In the generalized Kirchhoff boundary conditions, the wave functions have finite jumps across the vertex points and these jumps are compensated reciprocally in the sum of the first derivatives of the wave function. The Laplacian operator on the metric graphs with the generalized Kirchhoff boundary conditions is still extended to a self-adjoint operator similarly to the case with the classical Kirchhoff boundary conditions. Numerical confirmations of validity of the classical

Date: November 6, 2018.

This research was supported by the NSERC Discovery Grant. 
and generalized Kirchhoff boundary conditions are reported in a number of recent publications in physics literature [6, 25, 29].

In a series of papers [24, 26, 27], it was shown that if the parameters of the generalized Kirchhoff boundary conditions on a star graph are related to the parameters of the nonlinear evolution equations and satisfy a single constraint, then the nonlinear evolution equation on the star graph can be reduced to the homogeneous equation on the infinite line. In other words, singularities of the star graph are unfolded in the transformation and the vertex points become regular points on the line. In this case, a transmission of a solitary wave through the vertex points will be reflectionless.

The novelty of this paper is to explore the star graphs with the generalized Kirchhoff boundary conditions satisfying the constraint enabling reflectionless transmission of the solitary wave. We focus our study on the case example of the nonlinear Schrödinger (NLS) equation, which is the simplest nonlinear evolution equation considered on metric graphs [21]. In the simplest configuration of a star graph with a single vertex, we study existence and stability of stationary states of the NLS equation, which have a continuous parameter of their translations along the graph. We call such stationary states as the shifted states.

The shifted states of the NLS equation on a star graph appear naturally in the case of classical Kirchhoff boundary conditions when the number of edges is even. These states can be considered to be translations of the half-soliton states, which exist for any number of edges and the nonlinear instability of which was considered in our previous work [17] (see [1] for the very first prediction of nonlinear instability of the half-soliton states). In the variational characterization of the NLS stationary states on a star graph, such shifted states were mentioned in Remarks 5.3 and 5.4 in [2], where it was conjectured that all shifted states are saddle points of the action functional and are thus unstable for all star graphs with even number of edges exceeding two.

The purpose of this paper is to prove this conjecture with an explicit count of the Morse index for the shifted states. By extending the Sturm theory to Schrödinger operators on the star graph, we can give a very precise characterization of the negative and zero eigenvalues of the linearized Schrödinger operators, avoiding the less explicit theory of deficiency index for star graphs with point interactions [22]. As a result of our analysis, we prove that these shifted states are saddle points of energy subject to fixed mass, which are spectrally unstable under the NLS flow. In comparison, the half-soliton states are degenerate saddle points of energy and they are spectrally stable but nonlinearly unstable under the NLS flow.

We treat the star graph with an even number of edges as a particular example of the star graph with the generalized Kirchhoff boundary conditions satisfying the constraint enabling reflectionless transmission of the solitary wave [27]. In this more general context, we show that the shifted states satisfy the reduction of the NLS equation on the star graph to the homogeneous NLS equation on the infinite line. Nevertheless, we show that with one exception, the shifted states are spectrally unstable in the time evolution due to perturbations that break this reduction. Since numerical simulations have been performed in [24, 26, 27] with initial conditions satisfying this reduction and with solitary waves transmitted across the vertex points, no instability of the shifted states have been reported in the previous publications, to the best of our knowledge.

The only exception when the shifted states may be spectrally stable is the star graph with one incoming edge and $N-1$ outgoing edges. In this case, we prove that the shifted states with the monotone profiles in the $N-1$ outgoing edges are spectrally stable, whereas the shifted states with non-monotone profiles in the $N-1$ outgoing edges are spectrally unstable, the two families intersect at the nonlinearly unstable half-soliton state. In spite of the spectral stability of the shifted 
states with the monotone profiles, we give a simple argument that the shifted states are nonlinearly unstable due to perturbations that break homogenization of the NLS equation.

Our conjecture is that the central peak of the shifted state moves due to symmetry-breaking perturbations from the only incoming edge towards the vertex, breaks into $(N-1)$ peaks in the outgoing edges, the latter peaks become unstable due to spectral instability of the shifted states with the nonmonotone profiles. This conjecture is based on the comparison of the energy of the central peak in the incoming and outgoing edges at fixed mass. Due to this variational interpretation, spectral and nonlinear instabilities of the shifted states are likely to lead to a formation of a solitary wave escaping to infinity along one of the $(N-1)$ outgoing edges of the star graph. This dynamical picture appears to be in a complete agreement with the nonexistence results in [3] developed for the ground state of energy at fixed mass in the context of the star graphs with the classical Kirchhoff boundary conditions.

The paper is organized as follows. Section 2 gives a mathematical setup of the NLS equation on the star graph under the generalized Kirchhoff boundary conditions. Section 3 introduces the family of shifted states and discusses their properties. Main results on spectral stability of the shifted states are formulated in Section 4. The main results are proven in Section 5 with the count of the Morse index for the shifted states. Section 6 discusses homogenization of the star graph with the NLS equation on the infinite line. Variational interpretation of our results and our conjectures are described in Section 7.

\section{THE NLS EQUATION ON THE STAR GRAPH}

Let $\Gamma$ be a star graph, which consists of $N$ half-lines connected at a common vertex. The vertex is chosen as the origin and each edge of the star graph is parameterized by $\mathbb{R}^{+}$. The Hilbert space on the graph $\Gamma$ is given by

$$
L^{2}(\Gamma)=\oplus_{j=1}^{N} L^{2}\left(\mathbb{R}^{+}\right) .
$$

Elements in $L^{2}(\Gamma)$ are represented in the componentwise sense as vectors $\Psi=\left(\psi_{1}, \psi_{2}, \ldots, \psi_{N}\right)^{T}$ of $L^{2}\left(\mathbb{R}^{+}\right)$-functions with each component corresponding to one edge. The inner product and squared norm of such $L^{2}(\Gamma)$-functions are given by

$$
\langle\Psi, \Phi\rangle_{L^{2}(\Gamma)}:=\sum_{j=1}^{N} \int_{\mathbb{R}^{+}} \psi_{j}(x) \overline{\phi_{j}(x)} d x, \quad\|\Psi\|_{L^{2}(\Gamma)}^{2}:=\sum_{j=1}^{N}\left\|\psi_{j}\right\|_{L^{2}\left(\mathbb{R}^{+}\right)}^{2} .
$$

Similarly, we define the $L^{2}$-based Sobolev spaces on the graph $\Gamma$

$$
H^{k}(\Gamma)=\oplus_{j=1}^{N} H^{k}\left(\mathbb{R}^{+}\right), \quad k \in \mathbb{N}
$$

and equip them with suitable boundary conditions at the vertex.

For $k=1$, we set generalized continuity boundary conditions as follows:

$$
H_{\Gamma}^{1}:=\left\{\Psi \in H^{1}(\Gamma): \quad \alpha_{1}^{1 / p} \psi_{1}(0)=\alpha_{2}^{1 / p} \psi_{2}(0)=\cdots=\alpha_{N}^{1 / p} \psi_{N}(0)\right\},
$$

where $\alpha_{1}, \alpha_{2}, \ldots, \alpha_{N}$ are positive coefficients. These coefficients arise naturally when the onedimensional star graph is obtained as a limit of a narrow two-dimensional waveguide with different values of the thickness parameters that go to zero at the same rate [11, 12, 23].

For $k=2$, we set generalized Kirchhoff boundary conditions as follows:

$$
H_{\Gamma}^{2}:=\left\{\Psi \in H^{2}(\Gamma) \cap H_{\Gamma}^{1}: \quad \sum_{j=1}^{N} \frac{1}{\alpha_{j}^{1 / p}} \psi_{j}^{\prime}(0)=0\right\},
$$


where the prime stands for one-sided derivatives in $x$. The reason why the derivatives depend reciprocally on the positive coefficients $\alpha_{1}, \alpha_{2}, \ldots, \alpha_{N}$ is due to the requirement on the existence of a self-adjoint extension of the Laplacian operator in $L^{2}(\Gamma)$, as in the following lemma.

Lemma 2.1. There exists a self-adjoint extension of the Laplacian operator

$$
\Delta: H_{\Gamma}^{2} \subset L^{2}(\Gamma) \rightarrow L^{2}(\Gamma)
$$

Proof. If $\Psi \in H^{2}(\Gamma)$, then $\Psi(x), \Psi^{\prime}(x) \rightarrow 0$ as $x \rightarrow \infty$ by Sobolev embedding theorem. Therefore, for any $\Psi, \Phi \in H^{2}(\Gamma)$, integration by parts and boundary conditions in $(2.1)$ and $(2.2)$ yield

$$
\begin{aligned}
\langle\Delta \Psi, \Phi\rangle_{L^{2}(\Gamma)} & =\langle\Psi, \Delta \Phi\rangle_{L^{2}(\Gamma)}+\sum_{j=1}^{N} \psi_{j}(0) \bar{\phi}_{j}^{\prime}(0)-\sum_{j=1}^{N} \psi_{j}^{\prime}(0) \bar{\phi}_{j}(0) \\
& =\langle\Psi, \Delta \Phi\rangle_{L^{2}(\Gamma)}+\alpha_{1}^{1 / p} \psi_{1}(0) \sum_{j=1}^{N} \alpha_{j}^{-1 / p} \bar{\phi}_{j}^{\prime}(0)-\alpha_{1}^{1 / p} \bar{\phi}_{1}(0) \sum_{j=1}^{N} \alpha_{j}^{-1 / p} \psi_{j}^{\prime}(0) \\
& =\langle\Psi, \Delta \Phi\rangle_{L^{2}(\Gamma)} .
\end{aligned}
$$

By Theorem 1.4.4 in [5], the Laplacian operator $\Delta: L^{2}(\Gamma) \rightarrow L^{2}(\Gamma)$ with the domain $H_{\Gamma}^{2} \subset L^{2}(\Gamma)$ is extended to a self-adjoint operator.

The nonlinear Schrödinger (NLS) equation is posed on the star graph $\Gamma$ with the power nonlinearity:

$$
i \frac{\partial \Psi}{\partial t}=-\Delta \Psi-(p+1) \alpha^{2}|\Psi|^{2 p} \Psi, \quad x \in \Gamma, \quad t \in \mathbb{R}
$$

where $\Psi=\Psi(t, x)=\left(\psi_{1}, \psi_{2}, \ldots, \psi_{N}\right)^{T} \in \mathbb{C}^{N}, \Delta: L^{2}(\Gamma) \rightarrow L^{2}(\Gamma)$ is the Laplacian operator in Lemma 2.1, $\alpha \in L^{\infty}(\Gamma)$ is a piecewise constant function with the coefficients $\left(\alpha_{1}, \alpha_{2}, \ldots, \alpha_{N}\right) \in$ $\mathbb{R}_{+}^{N}$ defined on the edges of $\Gamma$, and the nonlinear term $\alpha^{2}|\Psi|^{2 p} \Psi$ is interpreted as a symbol for

$$
\left(\alpha_{1}^{2}\left|\psi_{1}\right|^{2 p} \psi_{1}, \alpha_{2}^{2}\left|\psi_{2}\right|^{2 p} \psi_{2}, \ldots, \alpha_{N}^{2}\left|\psi_{N}\right|^{2 p} \psi_{N}\right)^{T}
$$

The constant coefficients $\left(\alpha_{1}, \alpha_{2}, \ldots, \alpha_{N}\right)$ are the same as in the boundary conditions 2.1 and (2.2).

The NLS equation (2.3) is invariant under the gauge transformation $\Psi \mapsto e^{i \theta} \Psi$ and under the time translation $\Psi(t, x) \mapsto \Psi\left(t+t_{0}, x\right)$ with $\theta \in \mathbb{R}$ and $t_{0} \in \mathbb{R}$. The following lemma summarizes relevant results on the local-wellposedness of the Cauchy problem and on the conservation of the mass and energy functionals.

Lemma 2.2. For every $p>0$ and every $\Psi(0) \in H_{\Gamma}^{1}$, there exists $t_{0}>0$ and a local solution

$$
\Psi(t) \in C\left(\left(-t_{0}, t_{0}\right), H_{\Gamma}^{1}\right) \cap C^{1}\left(\left(-t_{0}, t_{0}\right), H^{-1}(\Gamma)\right)
$$

to the Cauchy problem associated with the NLS equation (2.3) such that the mass

$$
Q(\Psi):=\|\Psi\|_{L^{2}(\Gamma)}^{2},
$$

and the energy

$$
E(\Psi):=\left\|\Psi^{\prime}\right\|_{L^{2}(\Gamma)}^{2}-\left\|\alpha^{\frac{1}{p+1}} \Psi\right\|_{L^{2 p+2}(\Gamma)}^{2 p+2}
$$

are constant in $t \in\left(-t_{0}, t_{0}\right)$. 
Proof. Local well-posedness of the NLS equation 2.3 in $H_{\Gamma}^{1}$ is proved by using a standard contraction method thanks to the isometry of the semi-group $e^{i t \Delta}$ in $H_{\Gamma}^{1}$ and the Sobolev embedding of $H_{\Gamma}^{1}$ into $L^{\infty}(\Gamma)$.

Let us prove the mass and energy conservation under simplifying assumptions $p>1 / 2$ and $p \geq 1$ respectively. If $p>1 / 2$ and $\Psi(0) \in H_{\Gamma}^{2}$, it follows from the contraction method that there exists $t_{0}>0$ and a local strong solution

$$
\Psi(t) \in C\left(\left(-t_{0}, t_{0}\right), H_{\Gamma}^{2}\right) \cap C^{1}\left(\left(-t_{0}, t_{0}\right), L^{2}(\Gamma)\right)
$$

to the NLS equation 2.3. Applying time derivative to $Q(\Psi)$ and using the NLS equation (2.3) yield the mass balance equation:

$$
\begin{aligned}
\frac{d}{d t} Q(\Psi) & =-i\left\langle-\Delta \Psi-(p+1) \alpha^{2}|\Psi|^{2 p} \Psi, \Psi\right\rangle_{L^{2}(\Gamma)}+i\left\langle\Psi,-\Delta \Psi-(p+1) \alpha^{2}|\Psi|^{2 p} \Psi\right\rangle_{L^{2}(\Gamma)} \\
& =i\langle\Delta \Psi, \Psi\rangle_{L^{2}(\Gamma)}-i\langle\Psi, \Delta \Psi\rangle_{L^{2}(\Gamma)}=0
\end{aligned}
$$

where the last equality is obtained by Lemma 2.1. Thus, the mass conservation of 2.5 is proven for $\Psi(0) \in H_{\Gamma}^{2}$.

If $p>1 / 2$ and $\Psi(0) \in H_{\Gamma}^{1}$ but $\Psi(0) \notin H_{\Gamma}^{2}$, then in order to prove the mass conservation of 2.5., we define an approximating sequence $\left\{\Psi^{(n)}(0)\right\}_{n \in \mathbb{N}}$ in $H_{\Gamma}^{2}$ such that $\Psi^{(n)}(0) \rightarrow \Psi(0)$ in $H_{\Gamma}^{1}$ as $n \rightarrow \infty$. For each $\Psi^{(n)}(0) \in H_{\Gamma}^{2}$, there exists a local strong solution $\Psi^{(n)}(t)$ given by 2.7 for $t \in\left(-t_{0}^{(n)}, t_{0}^{(n)}\right)$. By Gronwall's inequality, there exists a positive constant $K$ which only depends on the $H^{1}(\Gamma)$ norm of the local solution $\Psi^{(n)}(t)$ such that

$$
\left\|\Psi^{(n) \prime \prime}(t)\right\|_{L^{2}(\Gamma)} \leq K\left\|\Psi^{(n) \prime \prime}(0)\right\|_{L^{2}(\Gamma)}, \quad t \in\left(-t_{0}^{(n)}, t_{0}^{(n)}\right),
$$

hence, the local existence time $t_{0}^{(n)}$ is determined by the $H^{1}(\Gamma)$ norm of the initial data $\Psi^{(n)}(0)$. Due to the convergence $\Psi^{(n)}(0) \rightarrow \Psi(0)$ in $H_{\Gamma}^{1}$, this implies that there is $t_{0}>0$ that depends on the $H^{1}(\Gamma)$ norm of $\Psi(0)$ such that $t_{0}^{(n)} \geq t_{0}$ for every $n \in \mathbb{N}$. Moreover, $\Psi^{(n)}(t) \rightarrow \Psi(t)$ in $H_{\Gamma}^{1}$ as $n \rightarrow \infty$ for every $t \in\left(-t_{0}, t_{0}\right)$. Since $Q\left(\Psi^{(n)}(t)\right)=Q\left(\Psi^{(n)}(0)\right)$ for every $t \in\left(-t_{0}, t_{0}\right)$, the limit $n \rightarrow \infty$ and the strong convergence in $H_{\Gamma}^{1}$ implies that $Q(\Psi(t))=Q(\Psi(0))$ for every $t \in\left(-t_{0}, t_{0}\right)$.

In order to prove the energy conservation, let us define the space $H_{\Gamma}^{3}$ compatible with the NLS flow:

$$
H_{\Gamma}^{3}:=\left\{\Psi \in H^{3}(\Gamma) \cap H_{\Gamma}^{2}: \quad \alpha_{1}^{1 / p} \psi_{1}^{\prime \prime}(0)=\alpha_{2}^{1 / p} \psi_{2}^{\prime \prime}(0)=\cdots=\alpha_{N}^{1 / p} \psi_{N}^{\prime \prime}(0)\right\}
$$

If $p \geq 1$ and $\Psi(0) \in H_{\Gamma}^{3}$, it follows from the contraction method that there exists $t_{0}>0$ and a local strong solution if $\Psi(0) \in H_{\Gamma}^{3}$

$$
\Psi(t) \in C\left(\left(-t_{0}, t_{0}\right), H_{\Gamma}^{3}\right) \cap C^{1}\left(\left(-t_{0}, t_{0}\right), H_{\Gamma}^{1}\right)
$$


to the NLS equation 2.3. Applying time derivative to $E(\Psi)$ and using the NLS equation (2.3) yield the energy balance equation:

$$
\begin{aligned}
\frac{d}{d t} E(\Psi)= & i\left\langle\Psi^{\prime \prime \prime}, \Psi^{\prime}\right\rangle_{L^{2}(\Gamma)}-i\left\langle\Psi^{\prime}, \Psi^{\prime \prime \prime}\right\rangle_{L^{2}(\Gamma)} \\
& +i(p+1)\left\langle\alpha^{2}\left(|\Psi|^{2 p}\right)^{\prime} \Psi, \Psi^{\prime}\right\rangle_{L^{2}(\Gamma)}-i(p+1)\left\langle\Psi^{\prime}, \alpha^{2}\left(|\Psi|^{2 p}\right)^{\prime} \Psi\right\rangle_{L^{2}(\Gamma)} \\
& +i(p+1)\left\langle\Psi^{p+1}, \alpha^{2} \Psi^{p} \Delta \Psi\right\rangle_{L^{2}(\Gamma)}-i(p+1)\left\langle\alpha^{2} \Psi^{p} \Delta \Psi, \Psi^{p+1}\right\rangle_{L^{2}(\Gamma)} \\
= & i \sum_{j=1}^{N} \psi_{j}^{\prime}(0)\left[\bar{\psi}_{j}^{\prime \prime}(0)+(p+1) \alpha_{j}^{2}\left|\psi_{j}(0)\right|^{2 p} \bar{\psi}_{j}(0)\right] \\
& -i \sum_{j=1}^{N} \bar{\psi}_{j}^{\prime}(0)\left[\psi_{j}^{\prime \prime}(0)+(p+1) \alpha_{j}^{2}\left|\psi_{j}(0)\right|^{2 p} \psi_{j}(0)\right],
\end{aligned}
$$

where the decay of $\Psi(x), \Psi^{\prime}(x)$, and $\Psi^{\prime \prime}(x)$ to zero at infinity has been used for the solution in $H_{\Gamma}^{3}$. Due to the boundary conditions in $\left.(2.1), \sqrt{2.2}\right)$, and $(2.8)$, we obtain $\frac{d}{d t} E(\Psi)=0$, that is, the energy conservation of 2.6 is proven for $\Psi(0) \in H_{\Gamma}^{3}$. The proof for $p \geq 1$ and $\Psi(0) \in H_{\Gamma}^{1}$ but $\Psi(0) \notin H_{\Gamma}^{3}$ is achieved by using an approximating sequence similarly to the argument above.

Finally, the proof can be extended to the local solution 2.4 for all values of $p>0$ by using other approximation techniques, see, e.g., Theorems 3.3.1, 3.3.5, and 3.39 in [7] or the proof of Proposition 2.2 in [2] for NLS on $\Gamma$ with $\alpha=1$.

Global existence in the NLS flow only holds in the subcritical case $p \in(0,2)$. In what follows, the scope of this work will be mainly developed in the subcritical case.

Lemma 2.3. For every $p \in(0,2)$, the local solution 2.4 in Lemma 2.2 is extended globally with $t_{0} \rightarrow \infty$.

Proof. This follows by the energy conservation and the Gagliardo-Nirenberg inequality

$$
\left\|\alpha^{\frac{1}{p+1}} \Psi\right\|_{L^{2 p+2}(\Gamma)}^{2 p+2} \leq C_{p, \alpha}\left\|\Psi^{\prime}\right\|_{L^{2}(\Gamma)}^{p}\|\Psi\|_{L^{2}(\Gamma)}^{p+2},
$$

for every $\alpha \in L^{\infty}(\Gamma), \Psi \in H_{\Gamma}^{1}, p>0$, where the constant $C_{p, \alpha}>0$ depends on $p$ and $\alpha$ but does not depend on $\Psi$.

In this work, we assume that the coefficients $\left(\alpha_{1}, \alpha_{2}, \ldots, \alpha_{N}\right)$ satisfy the following constraint:

$$
\sum_{j=1}^{K} \frac{1}{\alpha_{j}^{2 / p}}=\sum_{j=K+1}^{N} \frac{1}{\alpha_{j}^{2 / p}},
$$

where $K$ edges represent incoming bonds whereas the remaining $N-K$ edges represent outgoing bonds, clearly $K \neq 0$ and $K \neq N$. In particular, we consider two examples of the general star graph $\Gamma$ :

(i) $\alpha_{j}=1$ for all $j$ and $N$ is even. The boundary conditions in $H_{\Gamma}^{1}$ give the continuity at the vertex and the boundary conditions in $H_{\Gamma}^{2}$ are referred to as the classical Kirchhoff conditions. Constraint $(2.10)$ is satisfied with $K=N / 2$.

(ii) $K=1$ and $N \geq 3$. The graph $\Gamma$ consists of one incoming edge that splits into $N-1$ outgoing edges. The constraint (2.10) gives the reflectionless boundary conditions for the transmission of a solitary wave across the single vertex, as in [24, 26, 27]. 
Remark 2.4. If $N=2$, then the constraint (2.10) is only satisfied if $K=1$ and $\alpha_{1}=\alpha_{2}$. In this case, the NLS equation (6.5) on the graph $\Gamma$ is completely equivalent to the homogeneous NLS equation on the infinite line $\mathbb{R}$.

The stationary states of the NLS equation on the star graph $\Gamma$ under the constraint (2.10) include families parameterized by the translational parameter, as in the following section.

\section{STATIONARY STATES ON THE STAR GRAPH}

Stationary states of the NLS are given by the solutions of the form

$$
\Psi(t, x)=e^{i \omega t} \Phi_{\omega}(x),
$$

where $\left(\omega, \Phi_{\omega}\right) \in \mathbb{R} \times H_{\Gamma}^{2}$ is a real-valued solution of the stationary NLS equation,

$$
-\Delta \Phi_{\omega}-(p+1) \alpha^{2}\left|\Phi_{\omega}\right|^{2 p} \Phi_{\omega}=-\omega \Phi_{\omega}
$$

No solution $\Phi_{\omega} \in H_{\Gamma}^{2}$ to the stationary NLS equation $\sqrt{3.1}$ exist for $\omega \leq 0$ because $\sigma(-\Delta) \geq 0$ in $L^{2}(\Gamma)$ and $\Phi_{\omega}(x), \Phi_{\omega}^{\prime}(x) \rightarrow 0$ as $x \rightarrow \infty$ if $\Phi_{\omega} \in H_{\Gamma}^{2}$ by Sobolev's embedding theorems. For $\omega>0$, the scaling transformation $\Phi_{\omega}(x)=\omega^{\frac{1}{2 p}} \Phi(z)$ with $z=\omega^{\frac{1}{2}} x$ is employed to scale $\omega$ to unity. The normalized profile $\Phi \in H_{\Gamma}^{2}$ is now a solution of the stationary NLS equation

$$
-\Delta \Phi+\Phi-(p+1) \alpha^{2}|\Phi|^{2 p} \Phi=0 .
$$

For every $N$ and $\alpha$, the stationary NLS equation $(3.2)$ has a particular solution

$$
\Phi(x)=\left[\begin{array}{c}
\alpha_{1}^{-1 / p} \\
\alpha_{2}^{-1 / p} \\
\vdots \\
\alpha_{N}^{-1 / p}
\end{array}\right] \phi(x), \quad \text { with } \phi(x)=\operatorname{sech}^{\frac{1}{p}}(p x) .
$$

This solution is labeled in the previous paper [17] as the half-soliton state. In this work, we are interested in the families of solitary waves parameterized by a translational parameter, which are labeled as the shifted states. Such families exist if $\left(\alpha_{1}, \alpha_{2}, \ldots, \alpha_{N}\right)$ satisfy the constraint 2.10).

The following lemma gives the existence of a family of shifted states under the constraint (2.10).

Lemma 3.1. For every $p>0$ and every $\left(\alpha_{1}, \alpha_{2}, \ldots, \alpha_{N}\right)$ satisfying the constraint $(2.10)$, there exists a one-parameter family of solutions to the stationary NLS equation (3.2) with any $p>0$ given by $\Phi=\left(\phi_{1}, \ldots, \phi_{N}\right)^{T}$ with components

$$
\phi_{j}(x)= \begin{cases}\alpha_{j}^{-1 / p} \phi(x+a), & j=1, \ldots, K \\ \alpha_{j}^{-1 / p} \phi(x-a), & j=K+1, \ldots, N,\end{cases}
$$

where $\phi(x)=\operatorname{sech}^{\frac{1}{p}}(p x)$ and $a \in \mathbb{R}$ is arbitrary.

Proof. A general solution to the stationary NLS equation (3.2) decaying to zero at infinity is given by $\Phi=\left(\phi_{1}, \ldots, \phi_{N}\right)^{T}$ with components

$$
\phi_{j}(x)=\alpha_{j}^{-1 / p} \phi\left(x+a_{j}\right), \quad 1 \leq j \leq N,
$$


where $\left(a_{1}, \ldots, a_{N}\right) \in \mathbb{R}^{N}$ are arbitrary parameters. The continuity condition in $H_{\Gamma}^{2}$ imply that $\left|a_{1}\right|=\cdots=\left|a_{N}\right|$. In other words, for every $j=1, \ldots, N$, there exists $m_{j} \in\{0,1\}$, such that $a_{j}=(-1)^{m_{j}}|a|$ for some $a \in \mathbb{R}$. The Kirchhoff condition in $H_{\Gamma}^{2}$ is equivalent to

$$
\phi^{\prime}(|a|) \sum_{j=1}^{N} \frac{(-1)^{m_{j}}}{\alpha_{j}^{2 / p}}=0 .
$$

If $a=0$, the equation 3.5 holds since $\phi^{\prime}(0)=0$ and this yields the half-soliton state in the form (3.3). If $a \neq 0$, then the equation (3.5) holds due to the constraint $(2.10)$ if

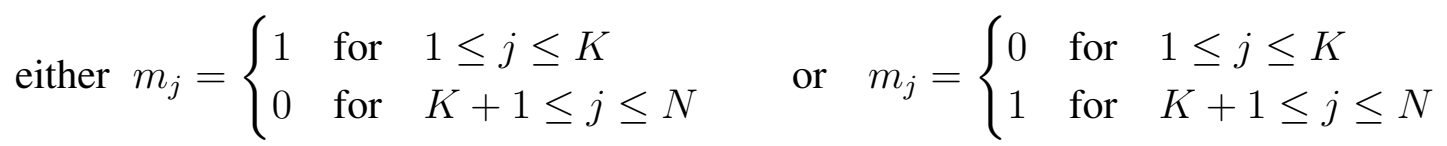

In both cases, the shifted state appears in the form (3.4) with either $a<0$ or $a>0$.

Remark 3.2. The half-soliton state (3.3) corresponds to the shifted state of Lemma 3.1 with $a=0$.

Remark 3.3. Besides the two choices specified in the proof of Lemma 3.1, there might be other $N$-tuples $\left(m_{1}, m_{2}, \ldots, m_{N}\right) \in\{0,1\}^{N}$ such that the bracket in $(3.5)$ becomes zero. Such $N$-tuples generate new one-parameter family different from the one given by Lemma 3.1 under the same constraint (2.10). For instance, if $\alpha_{j}=1$ for all $j$ and $K=N / 2$, there exist $C_{N}$ different shifted states given by Lemma 3.4 below with $C_{N}$ computed in (3.7).

The following lemma gives a full classification of families of shifted states in case (i) (see also Theorem 5 in [2]).

Lemma 3.4. For $\alpha=1$ and for even $N$, there exists $C_{N}$ one-parameter families of solutions to the stationary NLS equation (3.2) with any $p>0$, where

$$
C_{N}=\frac{N !}{2[(N / 2) !]^{2}} \text {. }
$$

Each family is generated from the simplest state $\Phi=\left(\phi_{1}, \ldots, \phi_{N}\right)^{T}$ with components

$$
\phi_{j}(x)= \begin{cases}\phi(x+a), & j=1, \ldots, \frac{N}{2} \\ \phi(x-a), & j=\frac{N}{2}+1, \ldots, N\end{cases}
$$

where $\phi(x)=\operatorname{sech}^{\frac{1}{p}}(p x)$ and $a \in \mathbb{R}$ is arbitrary, after rearrangements between $N / 2$ edges with $+a$ shifts and $N / 2$ edges with $-a$ shifts.

Proof. A general solution to the stationary NLS equation (3.2) decaying to zero at infinity is given by

$$
\Phi=\left(\phi\left(x+a_{1}\right), \ldots, \phi\left(x+a_{N}\right)\right)^{T},
$$

where $\phi(x)=\operatorname{sech}^{\frac{1}{p}}(p x)$, and $\left(a_{1}, \ldots, a_{N}\right) \in \mathbb{R}^{N}$ are arbitrary parameters. The continuity condition in $H_{\Gamma}^{2}$ imply that $\left|a_{1}\right|=\cdots=\left|a_{N}\right|$. The Kirchhoff condition in $H_{\Gamma}^{2}$ is equivalent to $\phi(a) \sum_{j=1}^{N} \tanh \left(a_{j}\right)=0$, which together with the continuity condition implies that the set $\left(a_{1}, \ldots, a_{N}\right)$ has exactly $\frac{N}{2}$ positive elements and exactly $\frac{N}{2}$ negative elements.

Remark 3.5. If $N=2$, then $C_{2}=1$. The only branch of shifted states in Lemma 3.4 corresponds to the NLS solitary wave translated along an infinite line $\mathbb{R}$, see Remark 2.4 
Remark 3.6. If $N=3$, then $C_{3}=3$. The three branches of shifted states in Lemma 3.4 correspond to the three possible NLS solitary waves translated along an infinite line $\mathbb{R}$, which is defined by the union of either $(1,2)$ or $(1,3)$, or $(2,3)$ edges of the star graph $\Gamma$.
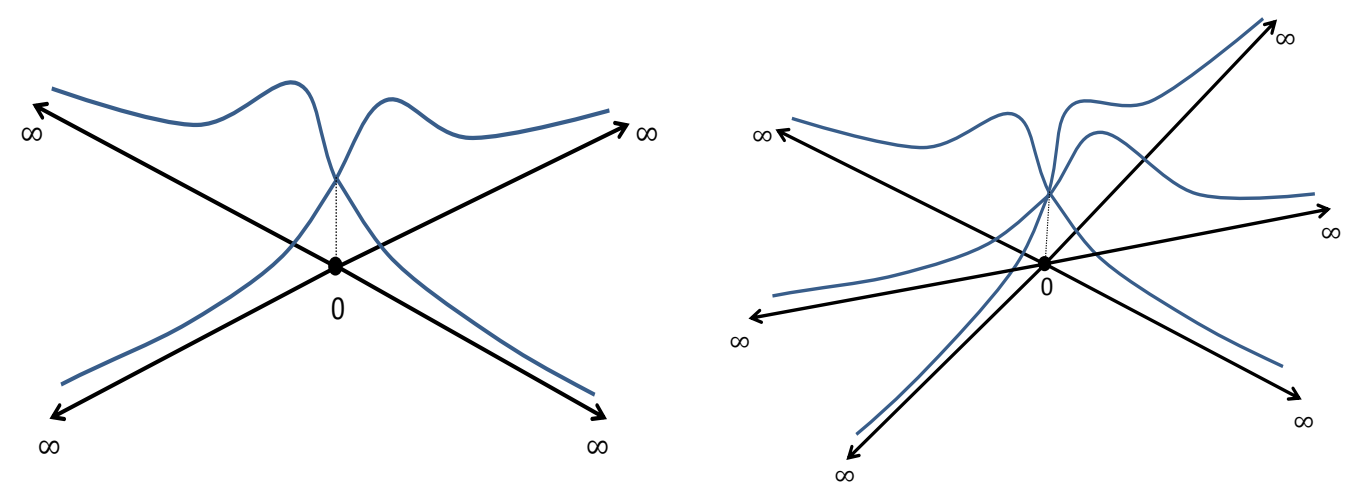

FIGURE 1. Schematic representation of the shifted states (3.8) with $a \neq 0$ for $N=4$ (left) and $N=6$ (right). The profile is monotonic in $N / 2$ edges and nonmonotonic in the other $N / 2$ edges.
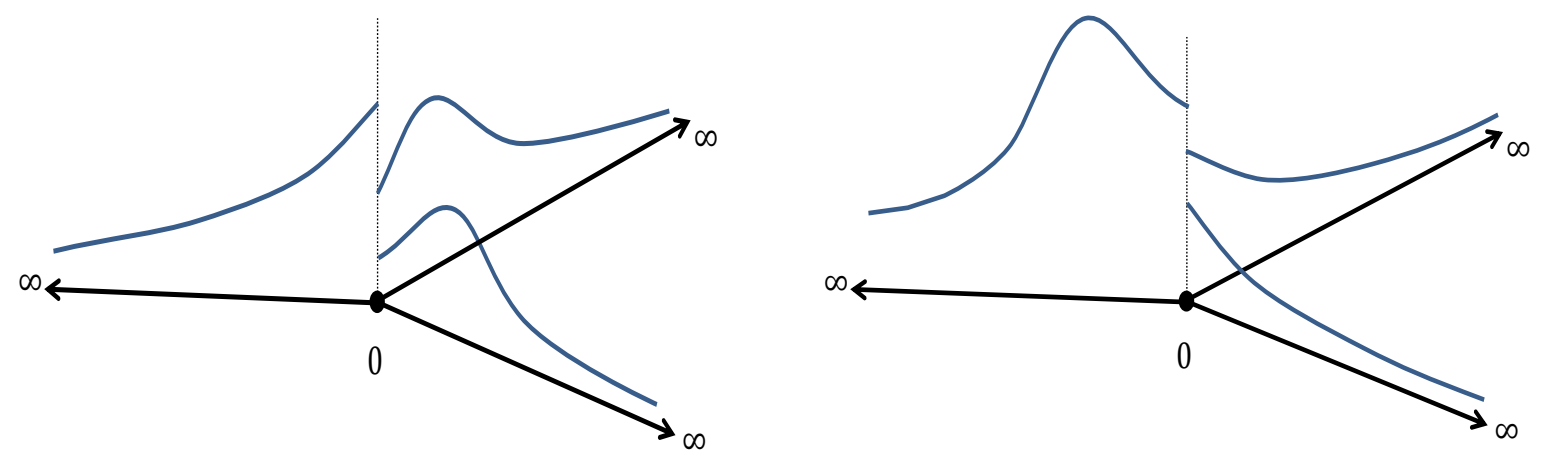

FIGURE 2. Schematic representation of the shifted states (3.4) with $K=1, N=3$, and either $a>0$ (left) or $a<0$ (right). For $a>0$, the profile is monotonic in 1 edge and non-monotonic in the other 2 edges, whereas for $a<0$, the situation is reversed.

For graphical illustrations, we present some of the shifted states on Figure 1 and 2 . Figure 1 shows shifted states in the case (i) with $N=4$ (left) and $N=6$ (right) when the shifted states are given by Lemma 3.4. If $a=0$, the profile of $\Phi$ contains $N / 2$ monotonic and $N / 2$ non-monotonic tails in different edges of the star graph $\Gamma$. Figures 2 shows shifted states in the case (ii) with $N=3$ when the shifted states are given by Lemma 3.1 with $K=1$. If $a>0$ (left), the profile of $\Phi$ contains 1 monotonic and 2 non-monotonic tails whereas if $a<0$ (right), the profile of $\Phi$ contains 2 monotonic and 1 non-monotonic tails. 


\section{MAin RESUltS ON STABILITY OF THE SHIFTED STATES}

Every stationary state $\Phi$ satisfying the stationary NLS equation (3.2) is a critical point of the action functional

$$
\Lambda(\Psi):=E(\Psi)+Q(\Psi), \quad \Psi \in H_{\Gamma}^{1},
$$

where $Q$ and $E$ are conserved mass and energy in $(2.5)$ and $(2.6)$ under the NLS flow, by Lemma 2.2. Substituting $\Psi=\Phi+U+i W$ with real-valued $U, W \in H_{\Gamma}^{1}$ into $\Lambda(\Psi)$ and expanding in $U, W$ yield

$$
\Lambda(\Phi+U+i W)=\Lambda(\Phi)+\left\langle L_{+} U, U\right\rangle_{L^{2}(\Gamma)}+\left\langle L_{-} W, W\right\rangle_{L^{2}(\Gamma)}+N(U, W)
$$

where

$$
\begin{aligned}
\left\langle L_{+} U, U\right\rangle_{L^{2}(\Gamma)} & :=\int_{\Gamma}\left[(\nabla U)^{2}+U^{2}-(2 p+1)(p+1) \alpha^{2} \Phi^{2 p} U^{2}\right] d x, \\
\left\langle L_{-} W, W\right\rangle_{L^{2}(\Gamma)} & :=\int_{\Gamma}\left[(\nabla W)^{2}+W^{2}-(p+1) \alpha^{2} \Phi^{2 p} W^{2}\right] d x
\end{aligned}
$$

and $N(U, W)=\mathrm{o}\left(\|U\|_{H^{1}(\Gamma)}^{2}+\|W\|_{H^{1}(\Gamma)}^{2}\right)$ for every $p>0$. The quadratic forms are defined by the two Hessian operators

$$
\begin{array}{llrl}
L_{+} & =-\Delta+1-(2 p+1)(p+1) \alpha^{2} \Phi^{2 p}: & H_{\Gamma}^{2} \subset L^{2}(\Gamma) \rightarrow L^{2}(\Gamma), \\
L_{-}= & -\Delta+1-(p+1) \alpha^{2} \Phi^{2 p}: & H_{\Gamma}^{2} \subset L^{2}(\Gamma) \rightarrow L^{2}(\Gamma),
\end{array}
$$

The number of negative eigenvalues of $L_{+}$and $L_{-}$is referred to as the Morse index of the shifted state $\Phi$. The following theorem represents the main result of this paper.

Theorem 4.1. Let $\Phi$ be a shifted state given by Lemma 3.1 with $a \neq 0$. Then $\sigma_{p}\left(L_{-}\right) \geq 0$ and 0 is a simple eigenvalue of $L_{-}$, whereas the non-positive part of $\sigma_{p}\left(L_{+}\right)$consists of a simple eigenvalue $\lambda_{0}<0$, another eigenvalue $\lambda_{1} \in\left(\lambda_{0}, 0\right)$ of multiplicity $K-1$ for $a<0$ and $N-K-1$ for $a>0$, and a simple zero eigenvalue. The rest of $\sigma_{p}\left(L_{-}\right)$and $\sigma_{p}\left(L_{+}\right)$is strictly positive and is bounded away from zero.

Remark 4.2. If $a=0$, it was established in our previous work [17] that the non-positive part of $\sigma_{p}\left(L_{+}\right)$for the half-solitons (3.3) consists of a simple eigenvalue $\lambda_{0}<0$ and a zero eigenvalue of multiplicity $N-1$.

By using the well-known results for the NLS equation [13] (see also [8]), we can deduce spectral instability of the shifted states from Theorem 4.1

Corollary 4.3. With the exception of case ( $i$ ) with $N=2$ or case (ii) with a $<0$, the shifted states with $a \neq 0$ are spectrally unstable in the time evolution of the NLS equation (2.3), in particular, there exists real positive eigenvalues $\lambda$ in the spectral stability problem

$$
\lambda\left[\begin{array}{c}
U \\
W
\end{array}\right]=\left[\begin{array}{cc}
0 & L_{-} \\
-L_{+} & 0
\end{array}\right]\left[\begin{array}{c}
U \\
W
\end{array}\right] .
$$

Moreover, with the account of algebraic multiplicity, for $p \in(0,2)$, there exist $K-1$ real positive eigenvalues $\lambda$ for $a<0$ and $N-K-1$ real positive eigenvalues for $a>0$. The shifted states of case (i) with $N=2$ or case (ii) with a $<0$ are spectrally stable for $p \in(0,2)$. 
Remark 4.4. The result of Theorem 4.1 and Corollary 4.3 in case (i) agrees with the qualitative picture described in Remark 5.3 in [2] and proves the conjecture formulated in Remark 5.4 in [2] that all shifted states (3.8) given by Lemma 3.4 are unstable for all even $N \geq 4$. If $N$ is even, the graph can be considered as a set of $K=N / 2$ copies of the real line and the shifted state can be interpreted as $K=N / 2$ identical solitary waves on each real line translated by the shift parameter $a \in \mathbb{R}$. Since $L_{-}$is positive at each solitary wave with a simple zero eigenvalue and $L_{+}$has a simple negative and a simple zero eigenvalue at each solitary wave, we can count $N / 2$ negative eigenvalues (with the account of their multiplicity), in agreement with the statement of Theorem 4.1. However, the multiplicity of the zero eigenvalue is not explained in this qualitative picture, and the count is incorrect for the half-soliton state (3.3), which corresponds to the case $a=0$, see Remark 3.2 .

Remark 4.5. Nonlinear instability of the half-soliton state (3.3) in case (i) with even $N \geq 4$ is proved in the previous paper [17] based on the characterization of the half-soliton state (3.3) as a degenerate saddle point of the action functional (4.1) under the constraint of fixed mass $Q$. The latter variational characterization was also given in [1]. The same argument holds for the half-soliton state (3.3) for any $N \geq 3$.

Remark 4.6. The difference between the normal form equations in [17] for odd and even $N$ clearly indicates the presence of shifted states in case $(i)$. The zero equilibrium state of the normal form equations is isolated for odd $N$, whereas it occurs at the intersection of $C_{N}$ lines of equilibria for even $N$, where $C_{N}$ is given by (3.7). In the latter case, for $N \geq 4$, transverse directions in the energy space to the $C_{N}$ lines of equilibria imply the saddle point geometry of the half-soliton state (3.3) as is proven in [17] for every $N \geq 3$.

Remark 4.7. For the two spectrally stable shifted states in Corollary 4.3 the case $(i)$ with $N=2$ is orbitally stable since the NLS equation on the star graph with $N=2$ is completely equivalent to the NLS equation on the infinite line $\mathbb{R}$. The case (ii) with a 0 is more challenging since the orbit of the shifted state has to be two-parametric due to phase rotation and translation in space, whereas the star graph $\Gamma$ is not equivalent to the real line $\mathbb{R}$ if $N \geq 3$. The translation in space can not be simply incorporated in the consideration because of the vertex point of the star graph. In Section 7, we give a simple argument why the shifted states (3.4) with a $<0$ are expected to be nonlinearly unstable under the NLS flow.

\section{COUnT OF THE MORSE INDEX FOR THE SHIFTED STATES}

In order to prove Theorem 4.1, we observe that the operators $L_{+}$and $L_{-}$defined in (4.3) and (4.4) are self-adjoint in $L^{2}(\Gamma)$. Since the bounded and exponentially decaying potential $\alpha^{2} \Phi^{2 p}$ is a relatively compact perturbation to the unbounded operator $L_{0}:=-\Delta+1$, the absolutely continuous spectra of $L_{ \pm}$, by Weyl's Theorem, is given by $\sigma_{c}\left(L_{ \pm}\right)=\sigma\left(L_{0}\right)=[1, \infty)$. Therefore, we are only concerned about the eigenvalues of $\sigma_{p}\left(L_{ \pm}\right)$in $(-\infty, 1)$.

Since $\Phi(x)>0$ for all $x \in \Gamma$, the same arguments as in Lemma 3.1 in [17] imply that $\sigma_{p}\left(L_{-}\right)$is nonnegative, $0 \in \sigma_{p}\left(L_{-}\right)$is a simple eigenvalue with the eigenvector $\Phi$, and all other eigenvalues in $\sigma_{p}\left(L_{-}\right)$are bounded away from zero. Hence we only need to consider $\sigma_{p}\left(L_{+}\right)$in $(-\infty, 1)$.

Spectral analysis involving various extensions of the operator $L_{+}$, Neumann formula, and the count of the deficiency index is used in [22] in a similar context. Instead of developing a functionalanalytic technique, we will prove the assertion of Theorem 4.1 about $\sigma_{p}\left(L_{+}\right)$by using Sturm's nodal count for the scalar Schrödinger equations. 
Sturm theory is well-known in the context of the Sturm-Liouville boundary-value problem on the finite interval (see, e.g., Section 5.1 in [28]). This theory was generalized for bounded graphs in a number of recent publications (see review in Section 5.2 in [5]). Extension of this theory to the unbounded graphs, e.g. to the star graph $\Gamma$, is not known to the best of author's knowledge. As an outcome of our work, we will show that the Sturm's nodal count is valid for the shifted states with $a \neq 0$ on the star graph $\Gamma$ in the same way as it is valid to the case of finite intervals or bounded graphs.

By using the representation (3.4), let us consider the exponentially decaying solutions of the second-order differential equation

(5.1) $-u^{\prime \prime}(x)+u(x)-(2 p+1)(p+1) \operatorname{sech}^{2}(p(x+a)) u(x)=\lambda u(x), \quad x \in(0, \infty), \quad \lambda<1$,

where $a \in \mathbb{R}$ is a parameter. By means of the substitution $u(x)=v(x+a)$ for $x \in(0, \infty)$, exponentially decaying solutions $u$ to the equation (5.1) are equivalent to exponentially decaying solutions $v$ of the second-order differential equation

$$
-v^{\prime \prime}(x)+v(x)-(2 p+1)(p+1) \operatorname{sech}^{2}(p x) v(x)=\lambda v(x), \quad x \in(a, \infty), \quad \lambda<1 .
$$

The following lemmas extend some well-known results on the scalar Schrödinger equation (5.2).

Lemma 5.1. For every $\lambda<1$, there exists a unique solution $v \in C^{1}(\mathbb{R})$ to equation (5.2) such that

$$
\lim _{x \rightarrow+\infty} v(x) e^{\sqrt{1-\lambda} x}=1 .
$$

Moreover, for any fixed $x_{0} \in \mathbb{R}, v\left(x_{0}\right)$ is a $C^{1}$ function of $\lambda$ for $\lambda<1$. The other linearly independent solution to equation (5.2) diverges as $x \rightarrow+\infty$.

Proof. The proof is based on the reformulation of the boundary-value problem (5.2)-(5.3) as Volterra's integral equation. By means of Green's function, the solution to (5.2) $-(5.3)$ can be found from the inhomogeneous integral equation

$$
v(x)=e^{-\sqrt{1-\lambda} x}-\frac{(2 p+1)(p+1)}{\sqrt{1-\lambda}} \int_{x}^{\infty} \sinh (\sqrt{1-\lambda}(x-y)) \operatorname{sech}^{2}(p y) v(y) \mathrm{d} y .
$$

Setting $w(x ; \lambda)=v(x) e^{\sqrt{1-\lambda} x}$ yields the following Volterra's integral equation with a bounded kernel:

$$
w(x ; \lambda)=1+\frac{(2 p+1)(p+1)}{2 \sqrt{1-\lambda}} \int_{x}^{\infty}\left(1-e^{-2 \sqrt{1-\lambda}(y-x)}\right) \operatorname{sech}^{2}(p y) w(y ; \lambda) \mathrm{d} y .
$$

By standard Neumann series, the existence and uniqueness of a bounded solution $w(\cdot ; \lambda) \in C^{1}\left(x_{0}, \infty\right)$ with $\lim _{x \rightarrow \infty} w(x ; \lambda)=1$ is obtained for every $\lambda<1$ and sufficiently large $x_{0} \gg 1$. By the ODE theory, this solution is extended globally as a solution $w(\cdot ; \lambda) \in C^{1}(\mathbb{R})$ of the integral equation (5.5). This construction yields a solution $v \in C^{1}(\mathbb{R})$ to the differential equation (5.2) with the exponential decay as $x \rightarrow+\infty$ given by (5.3). Since the Volterra's integral equation (5.4) depends analytically on $\lambda$ for $\lambda<1$, then $v\left(x_{0}\right)$ is (at least) $C^{1}$ function of $\lambda<1$ for any fixed $x_{0} \in \mathbb{R}$. Thanks to the $x$-independent and nonzero Wronskian determinant between two linearly independent solutions to the second-order equation (5.2), the other linearly independent solution diverges exponentially as $x \rightarrow+\infty$.

Lemma 5.2. Let $v$ be the solution defined in Lemma 5.1. If $v(0)=0$ (resp. $\left.v^{\prime}(0)=0\right)$ for some $\lambda_{0}<1$, then the corresponding eigenfunction $v$ to the Schrödinger equation (5.2) is an odd (resp. even) function on $\mathbb{R}$, whereas $\lambda_{0}$ is an eigenvalue of the associated Schrödinger operator defined 
in $L^{2}(\mathbb{R})$. There exists exactly one $\lambda_{0}<0$ corresponding to $v^{\prime}(0)=0$ and a simple eigenvalue $\lambda_{0}=0$ corresponding to $v(0)=0$, all other possible points $\lambda_{0}$ are located in $(0,1)$ bounded away from zero.

Proof. Extension of $v$ to an eigenfunction of the associated Schrödinger operator defined in $L^{2}(\mathbb{R})$ follows by the reversibility of the Schrödinger equation (5.2) with respect to the transformation $x \mapsto-x$. The count of eigenvalues follows by Sturm's Theorem since the odd eigenfunction for the eigenvalue $\lambda_{0}=0$,

$$
\phi^{\prime}(x)=-\operatorname{sech}^{\frac{1}{p}}(p x) \tanh (p x)
$$

has one zero on the infinite line. Hence, $\lambda_{0}=0$ is the second eigenvalue of the Schrödinger equation (5.2) with exactly one simple negative eigenvalue $\lambda_{0}<0$ that corresponds to an even eigenfunction.

Lemma 5.3. Let $v=v(x ; \lambda)$ be the solution defined by Lemma 5.1. Assume that $v\left(x ; \lambda_{1}\right)$ has a simple zero at $x=x_{1} \in \mathbb{R}$ for some $\lambda_{1} \in(-\infty, 1)$. Then, there exists a unique $C^{1}$ function $\lambda \mapsto x_{0}(\lambda)$ for $\lambda$ near $\lambda_{1}$ such that $v(x ; \lambda)$ has a simple zero at $x=x_{0}(\lambda)$ with $x_{0}\left(\lambda_{1}\right)=x_{1}$ and $x_{0}^{\prime}\left(\lambda_{1}\right)>0$.

Proof. By Lemma 5.1, $v$ is a $C^{1}$ function of $x$ and $\lambda$ for every $x \in \mathbb{R}$ and $\lambda \in(-\infty, 1)$. Since $x_{1}$ is a simple zero of $v\left(x ; \lambda_{1}\right)$, we have $\partial_{x} v\left(x_{1} ; \lambda_{1}\right) \neq 0$. By the implicit function theorem, there exists a unique $C^{1}$ function $\lambda \mapsto x_{0}(\lambda)$ for $\lambda$ near $\lambda_{1}$ such that $v(x ; \lambda)$ has a simple zero at $x=x_{0}(\lambda)$ with $x_{0}\left(\lambda_{1}\right)=x_{1}$. It remains to show that $x_{0}^{\prime}\left(\lambda_{1}\right)>0$.

Differentiating $v\left(x_{0}(\lambda) ; \lambda\right)=0$ in $\lambda$ at $\lambda=\lambda_{1}$, we obtain

$$
\partial_{x} v\left(x_{1} ; \lambda_{1}\right) x_{0}^{\prime}\left(\lambda_{1}\right)+\partial_{\lambda} v\left(x_{1} ; \lambda_{1}\right)=0 .
$$

Let us denote $\tilde{v}(x)=\partial_{\lambda} v\left(x ; \lambda_{1}\right)$. Differentiating equation 5.2 in $\lambda$ yields the inhomogeneous differential equation for $\tilde{v}$ :

$$
-\tilde{v}^{\prime \prime}(x)+\tilde{v}(x)-(2 p+1)(p+1) \operatorname{sech}^{2}(p x) \tilde{v}(x)=\lambda_{1} \tilde{v}(x)+v\left(x ; \lambda_{1}\right), \quad x \in(a, \infty), \quad \lambda<1 .
$$

By the same method based on the Volterra's integral equation as in Lemma 5.1, the function $\tilde{v}$ is $C^{1}$ in $x$ and decays to zero as $x \rightarrow \infty$. Therefore, by multiplying equation $(5.8)$ by $v\left(x ; \lambda_{1}\right)$, integrating by parts on $\left[x_{1}, \infty\right)$, and using equation $(5.2)$, we obtain

$$
-\partial_{x} v\left(x_{1} ; \lambda_{1}\right) \tilde{v}\left(x_{1}\right)=\int_{x_{1}}^{\infty} v\left(x ; \lambda_{1}\right)^{2} d x,
$$

where we have used $v\left(x_{1} ; \lambda_{1}\right)=0$ as well as the decay of $v\left(x ; \lambda_{1}\right), \partial_{x} v\left(x ; \lambda_{1}\right), \tilde{v}(x)$, and $\tilde{v}^{\prime}(x)$ to zero as $x \rightarrow \infty$. Combining (5.7) and (5.9) yields

$$
\left(\partial_{x} v\left(x_{1} ; \lambda_{1}\right)\right)^{2} x_{0}^{\prime}\left(\lambda_{1}\right)=\int_{x_{1}}^{\infty} v\left(x ; \lambda_{1}\right)^{2} d x>0,
$$

so that $x_{0}^{\prime}\left(\lambda_{1}\right)>0$ follows from the fact that $\partial_{x} v\left(x_{1} ; \lambda_{1}\right) \neq 0$.

The results of Lemmas 5.1, 5.2, and 5.3 are illustrated on Figure 3 which shows profile of the solution $v$ satisfying the limit (5.3) for four cases of $\lambda$ in $(-\infty, 0]$. The even eigenfunction for $\lambda_{0}<0$ and the odd eigenfunction for $\lambda=0$ correspond to the solutions of the Schrödinger equation defined in $L^{2}(\mathbb{R})$. The only zero $x_{0}(\lambda)$ of $v$ appears from negative infinity at $\lambda=\lambda_{0}$ and it is a monotonically increasing function of $\lambda$ in $\left(\lambda_{0}, 0\right)$ such that $x_{0}(0)=0$. 


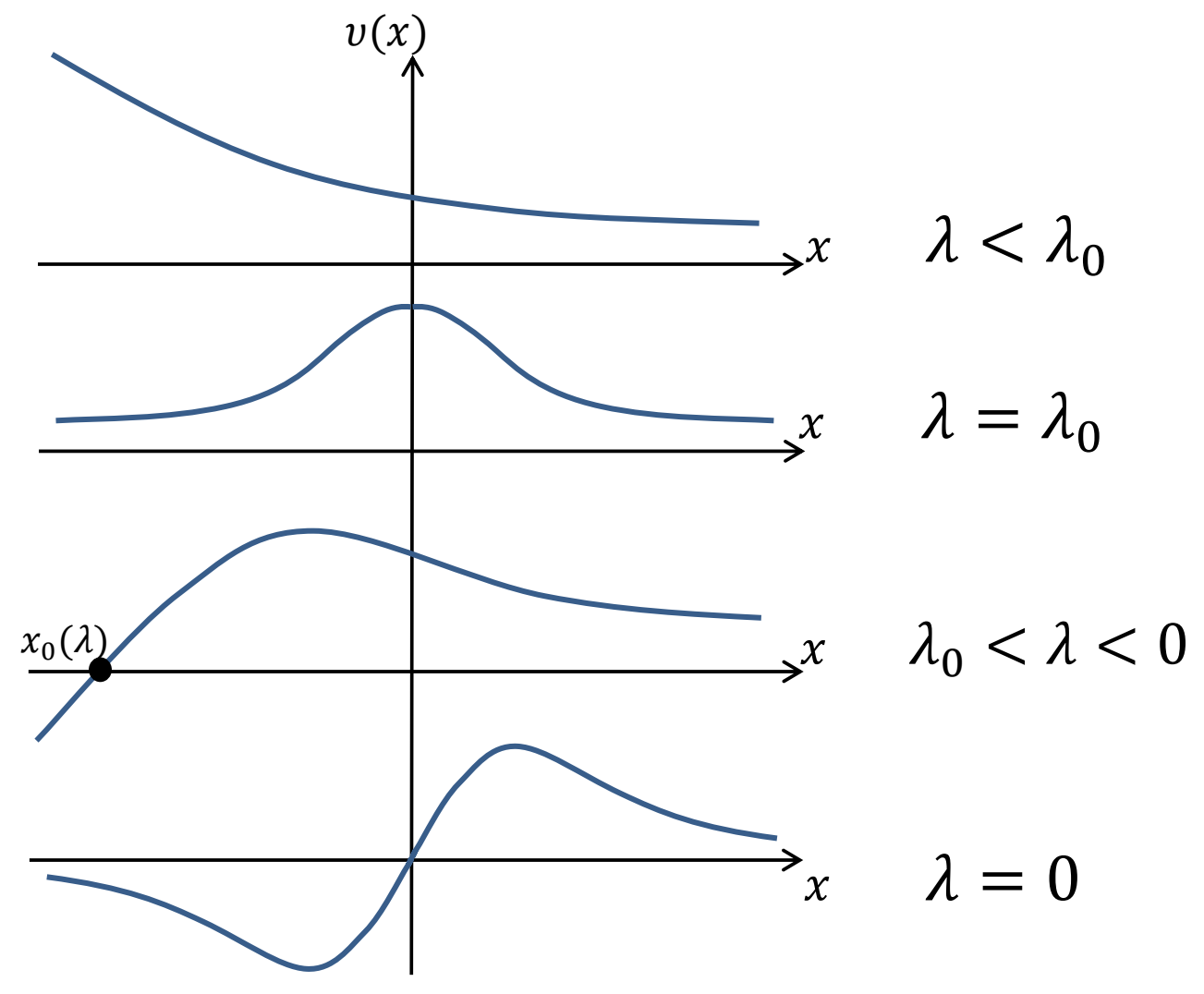

FIgURE 3. Profiles of the solution $v$ of the differential equation (5.2) with the limit (5.3).

By using the results of Lemmas 5.1, 5.2, and 5.3, we can address eigenvalues of $\sigma_{p}\left(L_{+}\right)$in $(-\infty, 1)$, where the differential expression for $L_{+}$is given by 4.3 .

Lemma 5.4. Let $v$ be the solution defined by Lemma 5.1. For every $a \in \mathbb{R}, \lambda_{0} \in(-\infty, 1)$ is an eigenvalue of $\sigma_{p}\left(L_{+}\right)$if and only if one of the following equations holds:

(a) $v(a)=0$

(b) $v(-a)=0$,

(c) $v(-a) v^{\prime}(a)+v(a) v^{\prime}(-a)=0$.

Moreover, $\lambda_{0} \in \sigma_{p}\left(L_{+}\right)$has multiplicity $K-1$ in the case (a), $N-K-1$ in the case (b), and is simple in the case (c). If $\lambda_{0}$ satisfies several cases, then its multiplicity is the sum of the multiplicities in each case.

Proof. Let $U \in H_{\Gamma}^{2}$ be the eigenvector of the operator $L_{+}$for the eigenvalue $\lambda_{0} \in \sigma_{p}\left(L_{+}\right)$. By Sobolev embedding of $H^{2}\left(\mathbb{R}^{+}\right)$into $C^{1}\left(\mathbb{R}^{+}\right)$, both $U(x)$ and $U^{\prime}(x)$ decay to zero as $x \rightarrow+\infty$. By using the representation (3.4) and the transformation of (5.1) to (5.2), we can write $U=$ $\left(u_{1}, \ldots, u_{N}\right)^{T}$ in the form

$$
u_{j}(x)= \begin{cases}c_{j} v(x+a), & j=1, \ldots, K, \\ c_{j} v(x-a), & j=K+1, \ldots, N,\end{cases}
$$


where $\left(c_{1}, c_{2}, \ldots, c_{N}\right)$ are coefficients and $v$ is the solution defined in Lemma 5.1. The boundary conditions for $U \in H_{\Gamma}^{2}$ in 2.1 and 2.2 imply the homogeneous linear system on the coefficients on $\left(c_{1}, c_{2}, \ldots, c_{N}\right)$ :

$$
c_{1} \alpha_{1}^{1 / p} v(a)=\cdots=c_{K} \alpha_{K}^{1 / p} v(a)=c_{K+1} \alpha_{K+1}^{1 / p} v(-a)=\cdots=c_{N} \alpha_{N}^{1 / p} v(-a)
$$

and

$$
\sum_{j=1}^{K} c_{j} \alpha_{j}^{-1 / p} v^{\prime}(a)+\sum_{j=K+1}^{N} c_{j} \alpha_{j}^{-1 / p} v^{\prime}(-a)=0 .
$$

The associated matrix is

$$
\left(\begin{array}{ccccccccc}
\alpha_{1}^{1 / p} v(a) & -\alpha_{2}^{1 / p} v(a) & 0 & \ldots & 0 & 0 & \ldots & 0 & 0 \\
\alpha_{1}^{1 / p} v(a) & 0 & -\alpha_{3}^{1 / p} v(a) & \ldots & 0 & 0 & \ldots & 0 & 0 \\
\vdots & \vdots & \vdots & \ddots & \vdots & \vdots & \ddots & \vdots & \vdots \\
\alpha_{1}^{1 / p} v(a) & 0 & 0 & \ldots & -\alpha_{K}^{1 / p} v(a) & 0 & \ldots & 0 & 0 \\
\alpha_{1}^{1 / p} v(a) & 0 & 0 & \ldots & 0 & -\alpha_{K+1}^{1 / p} v(-a) & \ldots & 0 & 0 \\
\vdots & \vdots & \vdots & \ddots & \vdots & \vdots & \ddots & \vdots & \vdots \\
\alpha_{1}^{1 / p} v(a) & 0 & 0 & \ldots & 0 & 0 & \ldots & 0 & -\alpha_{N}^{1 / p} v(-a) \\
b_{1} & b_{2} & b_{3} & \ldots & b_{K} & b_{K+1} & \ldots & b_{N-1} & b_{N}
\end{array}\right)
$$

where

$$
b_{j}=\left\{\begin{array}{l}
\alpha_{j}^{-1 / p} v^{\prime}(a), \quad 1 \leq j \leq K \\
\alpha_{j}^{-1 / p} v^{\prime}(-a), \quad K+1 \leq j \leq N .
\end{array}\right.
$$

In order to calculate the determinant of the associate matrix, we perform elementary column operations and obtain a lower triangular matrix. Let the associate matrix be of the form $\left[A_{1}^{0} A_{2}^{0} \ldots A_{N}^{0}\right]$, where $A_{j}^{0}$ represents the $j$-th column of the matrix in the beginning of the algorithm. Then, we perform the following elementary column operations:

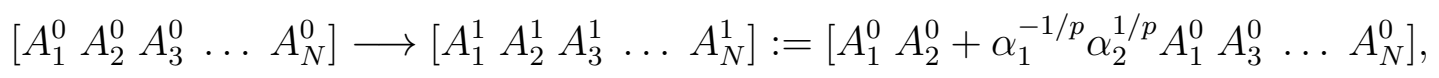

then

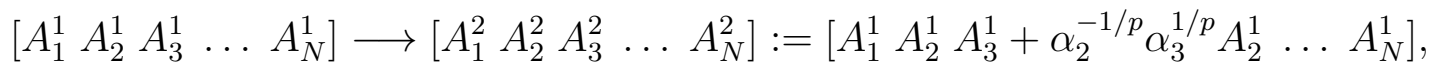

and so on, until the $K$-th step. At the $K$-th step, we need to take into account the change of $v(a)$ to $v(-a)$ in the $K+1$-th column, hence the $K$-th step involves

$$
A_{K+1}^{K-1} \longrightarrow A_{K+1}^{K}:=A_{K+1}^{K-1}+\frac{\alpha_{K+1}^{1 / p} v(-a)}{\alpha_{K}^{1 / p} v(a)} A_{K}^{K-1} .
$$

At the $(K+1)$-th and subsequent steps, no further changes of $v(-a)$ occurs, so that we apply the same rule as the one before the $K$-th step in all subsequent transformations. Finally, after $(N-1)$ 
transformations, we obtain a lower triangular matrix in the form:

$$
\left(\begin{array}{ccccccccc}
\alpha_{1}^{1 / p} v(a) & 0 & 0 & \ldots & 0 & 0 & \ldots & 0 & 0 \\
\alpha_{1}^{1 / p} v(a) & \alpha_{2}^{1 / p} v(a) & 0 & \ldots & 0 & 0 & \ldots & 0 & 0 \\
\alpha_{1}^{1 / p} v(a) & \alpha_{2}^{1 / p} v(a) & \alpha_{3}^{1 / p} v(a) & \ldots & 0 & 0 & \ldots & 0 & 0 \\
\vdots & \vdots & \vdots & \ddots & \vdots & \vdots & \ddots & \vdots & \vdots \\
\alpha_{1}^{1 / p} v(a) & \alpha_{2}^{1 / p} v(a) & \alpha_{3}^{1 / p} v(a) & \ldots & \alpha_{K}^{1 / p} v(a) & 0 & \ldots & 0 & 0 \\
\alpha_{1}^{1 / p} v(a) & \alpha_{2}^{1 / p} v(a) & \alpha_{3}^{1 / p} v(a) & \ldots & \alpha_{K}^{1 / p} v(a) & \alpha_{K+1}^{1 / p} v(-a) & \ldots & 0 & 0 \\
\vdots & \vdots & \vdots & \ddots & \vdots & \vdots & \ddots & \vdots & \vdots \\
\alpha_{1}^{1 / p} v(a) & \alpha_{2}^{1 / p} v(a) & \alpha_{3}^{1 / p} v(a) & \ldots & \alpha_{K}^{1 / p} v(a) & \alpha_{K+1}^{1 / p} v(-a) & \ldots & \alpha_{N-1}^{1 / p} v(-a) & 0 \\
B_{1} & B_{2} & B_{3} & \ldots & B_{K} & B_{K+1} & \ldots & B_{N-1} & B_{N}
\end{array}\right)
$$

where $\left\{B_{j}\right\}_{j=1}^{N}$ are some numerical coefficients, in particular, $B_{1}=\alpha_{1}^{-1 / p} v^{\prime}(a)$ and

$$
B_{N}=\frac{\alpha_{N}^{1 / p}}{v(a)}\left[\sum_{j=1}^{K} \alpha_{j}^{-2 / p} v^{\prime}(a) v(-a)+\sum_{j=K+1}^{N} \alpha_{j}^{-2 / p} v^{\prime}(-a) v(a)\right]
$$

Under the constraint (2.10), the determinant of the lower triangular matrix is evaluated in the form:

$$
\Delta=\left(\prod_{j=1}^{N} \alpha_{j}^{1 / p}\right)\left(\sum_{j=1}^{K} \alpha_{j}^{-2 / p}\right) v(a)^{K-1} v(-a)^{N-K-1}\left[v(-a) v^{\prime}(a)+v(a) v^{\prime}(-a)\right] .
$$

Therefore, $U \neq 0$ is the eigenvector of $L_{+}$for the eigenvalue $\lambda_{0} \in(-\infty, 1)$ if and only if $\Delta=0$, or equivalently, if either $v(a)=0$ or $v(-a)=0$ or $v(-a) v^{\prime}(a)+v(a) v^{\prime}(-a)=0$.

In the case of $v(a)=0$ and $v(-a) \neq 0$, it follows from the linear system $(5.11)$ that $c_{j}=0$ for all $K+1 \leq j \leq N$ and $c_{j} \in \mathbb{R}$ are arbitrary for all $1 \leq j \leq K$. The linear equation (5.12) implies that $\sum_{j=1}^{K} c_{j} \alpha_{j}^{-1 / p}=0$, since $v^{\prime}(a) \neq 0$ when $v(a)=0$. Therefore, the eigenvalue $\lambda_{0}$ has a multiplicity $K-1$.

Similarly, the eigenvalue $\lambda_{0}$ has a multiplicity $N-K-1$ if $v(a) \neq 0$ and $v(-a)=0$.

In the case $v(-a) v^{\prime}(a)+v(a) v^{\prime}(-a)=0$ but $v(a) \neq 0$ and $v(-a) \neq 0$, the linear system (5.11) implies that all coefficients are related to one coefficient. The linear equation (5.12) is then satisfied due to the constraint (2.10) and $\lambda_{0}$ is a simple eigenvalue.

If several cases are satisfied simultaneously, then it follows from the linear system (5.11) and (5.12) that multiplicity of $\lambda_{0}$ is equal to the sum of the multiplicities for each of the cases.

Proof of Theorem 4.1. The result on $\sigma_{p}\left(L_{-}\right)$is proved from Lemma 3.1 in [17]. The construction of $\sigma_{p}\left(L_{+}\right)$follows from Lemmas 5.2, 5.3, and 5.4, as well as the continuation arguments.

The condition (c) in Lemma 5.4 is satisfied if the solution $v$ in Lemma 5.1 is either odd or even function of $a$. For the simple eigenvalue $\lambda_{0}<0$ in Lemma 5.2, the eigenfunction is even and positive. Hence, $v(a) \neq 0$ and $v(-a) \neq 0$, so that $\lambda_{0}$ is a simple eigenvalue in $\sigma_{+}\left(L_{+}\right)$by the case (c) in Lemma 5.4. The corresponding eigenvector $U \in H_{\Gamma}^{2}$ is strictly positive definite on $\Gamma$.

For the simple zero eigenvalue in Lemma 5.2, the eigenfunction (5.6) is odd and positive on $(-\infty, 0)$. Since $v(a) \neq 0$ and $v(-a) \neq 0$ if $a \neq 0$. then 0 is a simple eigenvalue in $\sigma_{+}\left(L_{+}\right)$by the 
case (c) in Lemma 5.4. The corresponding eigenvector $U \in H_{\Gamma}^{2}$ can be represented in the form:

$$
U(x)=\left\{\begin{array}{ll}
\alpha_{j}^{-1 / p} \phi^{\prime}(x+a), & j=1, \ldots, K \\
-\alpha_{j}^{-1 / p} \phi^{\prime}(x-a), & j=K+1, \ldots, N
\end{array} .\right.
$$

which represent the translation of the shifted state (3.4) with respect to parameter $a$.

No other values of $\lambda_{0}$ exists in $\left(-\infty, \lambda_{2}\right)$ such that the condition (c) in Lemma 5.4 is satisfied, where $\lambda_{2}>0$ is either the positive eigenvalue of the scalar Schrödinger equation (5.2) or the bottom of $\sigma_{c}\left(L_{+}\right)$at $\lambda_{2}=1$.

If $a>0$, then we claim that $v(a)>0$ for every $\lambda \in(-\infty, 0]$. Indeed, by Lemma 5.3, simple zeros of $v$ are monotonically increasing functions of $\lambda$, whereas no multiple zeros of $v$ may exist for nonzero solutions of the second-order differential equations. Since the only zero of $v$ bifurcates from $x=-\infty$ at $\lambda=\lambda_{0}<0$ and reaches $x=0$ at $\lambda=0, v(x)$ remains positive for every $x>0$ for $\lambda \in(-\infty, 0]$. Hence the condition (a) in Lemma 5.4 is not satisfied for every $\lambda \in(-\infty, 0]$.

We now consider vanishing of $v(-a)$ for $a>0$ for the condition (b) in Lemma 5.4. By the same continuation argument from Lemma 5.3, there exists exactly one $\lambda_{1} \in\left(\lambda_{0}, 0\right)$ such that $v(-a)=0$ for any given $a>0$. Since $v^{\prime}(-a) \neq 0$ and $v(a) \neq 0, \lambda_{1}$ is an eigenvalue of $\sigma_{p}\left(L_{+}\right)$of multiplicity $N-K-1$.

For $a<0$, the roles of cases (a) and (b) are swaped. The condition (b) is never satisfied, while the condition (a) is satisfied for exactly one $\lambda_{1} \in\left(\lambda_{0}, 0\right)$, which becomes an eigenvalue of $\sigma_{p}\left(L_{+}\right)$ of multiplicity $K-1$. The assertion of Theorem 4.1 is proved.

Remark 5.5. For $p=1$, the solution $v$ in Lemma 5.1 is available in the closed analytic form:

$$
v(x)=e^{-\sqrt{1-\lambda} x} \frac{3-\lambda+3 \sqrt{1-\lambda} \tanh x-3 \operatorname{sech}^{2} x}{3-\lambda+3 \sqrt{1-\lambda}} .
$$

In this case, $\lambda_{0}=-3$ is a simple eigenvalue corresponding to $v(x)=\frac{1}{4} \operatorname{sech}^{2} x$ and 0 is a simple eigenvalue corresponding to $v(x)=\frac{1}{2} \tanh x \operatorname{sech} x$. If $a \neq 0$, the negative eigenvalue $\lambda_{1} \in\left(\lambda_{0}, 0\right)$ in the proof of Theorem 4.1 is given by the root of the following transcendental equation

$$
3-\lambda-3 \sqrt{1-\lambda} \tanh |a|-3 \operatorname{sech}^{2}(a)=0,
$$

or explicitly, by

$$
\lambda_{1}=-\frac{3}{2} \tanh |a|\left[\tanh |a|+\sqrt{1+3 \operatorname{sech}^{2}(a)}\right],
$$

We note that $\lambda_{1} \rightarrow 0$ when $a \rightarrow 0$ and $\lambda_{1} \rightarrow \lambda_{0}=-3$ when $|a| \rightarrow \infty$.

Remark 5.6. By the count of Theorem 4.1 the Morse index of $L_{+}$is $K$ if a $<0$ and $N-K$ if $a>0$. On the other hand, the Sturm index (defined as the number of nodes for the eigenfunction $U$ in (5.13) corresponding to the eigenvalue $\lambda=0)$ is $K$ if $a<0$ and $N-K$ if $a>0$. Hence, the two indices are equal to each other, similarly to the Sturm's nodal count for finite intervals or bounded graphs.

\section{HOMOGENIZATION OF THE STAR GRAPH}

The translational symmetry in the star graph $\Gamma$ is broken due to the vertex at $x=0$. As a result, a momentum functional is not generally conserved under the NLS flow. However, we will show 
here that if the coefficients $\left(\alpha_{1}, \alpha_{2}, \ldots, \alpha_{N}\right)$ satisfy the constraint $(2.10)$, then there exist solutions to the NLS equation 2.3 , for which the following momentum functional is conserved:

$$
P(\Psi):=\sum_{j=1}^{N}(-1)^{m_{j}} \int_{\mathbb{R}^{+}} \operatorname{Im}\left(\psi_{j}^{\prime} \bar{\psi}_{j}\right) d x,
$$

where the $N$-tuple $\left(m_{1}, m_{2}, \ldots, m_{N}\right)$ is given by $(3.6)$. Computations yields the following momentum balance equation.

Lemma 6.1. For every $p>0$ and every $\left(\alpha_{1}, \alpha_{2}, \ldots, \alpha_{N}\right)$ satisfying the constraint $(2.10)$, the local solution (2.4) in Lemma 2.2 satisfies the momentum balance equation

$$
\frac{d}{d t} P(\Psi)=\sum_{j=1}^{N}(-1)^{m_{j}}\left|\psi_{j}^{\prime}(0)\right|^{2} .
$$

for all $t \in\left(-t_{0}, t_{0}\right)$, where $P$ is given by $(6.1)$.

Proof. If $p \geq 1$, we can consider the smooth solutions (2.9) to the NLS equation (2.3). The momentum balance equation for $P$ in (6.1) can be written in the form:

$$
\frac{d}{d t} P(\Psi)=\sum_{j=1}^{N}(-1)^{m_{j}} \int_{\mathbb{R}^{+}} \operatorname{Im}\left(\psi_{j}^{\prime} \partial_{t} \bar{\psi}_{j}+\bar{\psi}_{j} \partial_{t} \psi_{j}^{\prime}\right) d x .
$$

By using the formulas for $\partial_{t} \psi_{j}$ and $\partial_{t} \psi_{j}^{\prime}$ obtained from the NLS equation $\sqrt{2.3}$, we can simplify the momentum balance equation (6.3) and verify the following chain of equations with the integration by parts technique:

$$
\begin{aligned}
\frac{d}{d t} P(\Psi) & =\sum_{j=1}^{N}(-1)^{m_{j}} \int_{\mathbb{R}^{+}} \operatorname{Re}\left(\bar{\psi}_{j} \psi_{j}^{\prime \prime \prime}-\psi_{j}^{\prime} \bar{\psi}_{j}^{\prime \prime}+p \alpha_{j}^{2}\left(\left|\psi_{j}\right|^{2 p+2}\right)^{\prime}\right) d x \\
& =\sum_{j=1}^{N}(-1)^{m_{j}}\left(-\operatorname{Re}\left[\bar{\psi}_{j}(0) \psi_{j}^{\prime \prime}(0)\right]+\left|\psi_{j}^{\prime}(0)\right|^{2}-p \alpha_{j}^{2}\left|\psi_{j}(0)\right|^{2 p+2}\right),
\end{aligned}
$$

where the decay of $\Psi(x), \Psi^{\prime}(x)$, and $\Psi^{\prime \prime}(x)$ to zero at infinity has been used for the solution in $H_{\Gamma}^{3}$. Applying the boundary conditions in (2.1) and (2.8), the constraint (2.10), and the choice of values of $m_{j}$ in (3.6) yields the momentum balance equation in the form (6.2).

Although our derivation was restricted to the case $p \geq 1$ and to solutions in $H_{\Gamma}^{3}$, the proof can be extended to the local solution $(2.4)$ for all values of $p>0$ by using standard approximation techniques [7].

The momentum $P(\Psi)$ is conserved in $t$ if the boundary conditions for derivatives satisfy the additional constraints:

$$
(-1)^{m_{1}} \alpha_{1}^{1 / p} \psi_{1}^{\prime}(0)=(-1)^{m_{2}} \alpha_{2}^{1 / p} \psi_{2}^{\prime}(0)=\cdots=(-1)^{m_{N}} \alpha_{N}^{1 / p} \psi_{N}^{\prime}(0),
$$

which are compatible with the boundary conditions in (2.2) under the constraint (2.10). Under the constraint (6.4), we verify from equation (6.2) that

$$
\frac{d}{d t} P(\Psi)=(-1)^{m_{1}} \alpha_{1}^{2 / p}\left|\psi_{1}^{\prime}(0)\right|^{2}\left(\sum_{j=1}^{K} \frac{1}{\alpha_{j}^{2 / p}}-\sum_{j=K+1}^{N} \frac{1}{\alpha_{j}^{2 / p}}\right)=0,
$$


hence $P(\Psi)$ is conserved in $t$.

In order to make sure that the constraint (6.4) is satisfied for every $t$, we observe the following reduction of the NSL equation (2.3) on the star graph $\Gamma$ to the homogeneous NLS equation on the infinite line.

Lemma 6.2. Under the constraint (2.10), there exist solutions of the NLS equation (2.3) on the graph $\Gamma$ which satisfy the the following homogeneous NLS equation on the infinite line:

$$
i U_{t}+U_{x x}+(p+1)|U|^{2 p} U=0, \quad x \in \mathbb{R}, \quad t \in \mathbb{R},
$$

where $U=U(t, x) \in \mathbb{C}$.

Proof. The class of suitable solutions $\Psi$ to the NLS equation 2.3 on the star graph $\Gamma$ must satisfy the following reduction:

$$
\left\{\begin{array}{l}
\alpha_{1}^{1 / p} \psi_{1}(t, x)=\cdots=\alpha_{K}^{1 / p} \psi_{K}(t, x), \\
\alpha_{K+1}^{1 / p} \psi_{K+1}(t, x)=\cdots=\alpha_{N}^{1 / p} \psi_{N}(t, x),
\end{array} \quad x \in \mathbb{R}^{+}, \quad t \in \mathbb{R},\right.
$$

subject to the boundary conditions at the vertex point $x=0$ :

$$
\alpha_{K}^{1 / p} \psi_{K}(t, 0)=\alpha_{K+1}^{1 / p} \psi_{K+1}(t, 0), \quad \alpha_{K}^{1 / p} \partial_{x} \psi_{K}(t, 0)=-\alpha_{K+1}^{1 / p} \partial_{x} \psi_{K+1}(t, 0) .
$$

Note that the boundary conditions are compatible with the generalized Kirchhoff boundary conditions in (2.2) under the constraint (2.10). Thanks to the reduction (6.6), the following function can be defined on the infinite line:

$$
U(t, x):= \begin{cases}\alpha_{j}^{1 / p} \psi_{j}(t,-x), \quad 1 \leq j \leq K, & x \in \mathbb{R}^{-}, \\ \alpha_{j}^{1 / p} \psi_{j}(t, x), \quad K+1 \leq j \leq N, & x \in \mathbb{R}^{+} .\end{cases}
$$

Thanks to the boundary conditions 6.7, $U$ is a $C^{1}$ function across $x=0$. Substitution 6.8 into the NLS equation (2.3) on the graph $\Gamma$ yields the homogeneous NLS equation (6.5), where the point $x=0$ is a regular point on the infinite line $\mathbb{R}$.

Remark 6.3. The shifted state (3.4) corresponds to the NLS soliton in the homogeneous NLS equation (6.5), which is translational invariant along the line $\mathbb{R}$. The eigenvalue count of Theorem 4.1 and the instability result of Corollary 4.3 are related to the symmetry-breaking perturbations, which do not satisfy the reduction (6.6). These perturbations satisfy the NLS equation (2.3) on the graph $\Gamma$ but do not satisfy the homogeneous NLS equation (6.5) on the line $\mathbb{R}$. Such symmetrybreaking perturbations were not considered in [24, 26, 27].

\section{VARIATIONAL CHARACTERIZATION OF THE SHIFTED STATES}

Here we give a simple argument suggesting that the spectrally stable shifted states in the case (ii) with $K=1$ and $a<0$ are nonlinearly unstable under the NLS flow. This involves the variational characterization of the shifted states in the graph $\Gamma$ as critical points of energy under the fixed mass, where the mass and energy are defined by (2.5) and (2.6) respectively.

The mass and energy are computed at the shifted states (3.4) as follows:

$$
Q(\Phi)=\left(\sum_{j=1}^{K} \alpha_{j}^{-2 / p}\right)\|\phi\|_{L^{2}(\mathbb{R})}^{2}
$$


and

$$
E(\Phi)=\left(\sum_{j=1}^{K} \alpha_{j}^{-2 / p}\right)\left(\left\|\phi^{\prime}\right\|_{L^{2}(\mathbb{R})}^{2}-\|\phi\|_{L^{2 p+2}(\mathbb{R})}^{2 p+2}\right),
$$

where the constraint 2.10) has been used. In the case (ii) with $K=1$, the mass and energy at the shifted states is the same as the mass and energy of a free solitary wave escaping to infinity along the incoming edge. This property signals out that the infimum of energy is not achieved, as is discussed in [3].

Furthermore, the constraint 2.10) implies that $\alpha_{2}, \ldots, \alpha_{N}>\alpha_{1}$ (if $N \geq 3$ ). Pick the $j$-th outgoing edge for $2 \leq j \leq N$ and fix the mass at the level $\mu>0$. Then, it is well-known [3] that the energy of a free solitary wave escaping to infinity along the $j$-th outgoing edge is given by

$$
E_{j}=-C_{p} \alpha_{j}^{\frac{4}{2-p}} \mu^{\frac{p+2}{2-p}}<-C_{p} \alpha_{1}^{\frac{4}{2-p}} \mu^{\frac{p+2}{2-p}}=E(\Phi)
$$

where $p \in(0,2)$ and $C_{p}$ is an universal constant that only depends on $p$. Thus, a free solitary wave escaping the graph $\Gamma$ along any outgoing edge has a lower energy level at fixed mass compared to the shifted state. This suggests that any shifted state is energetically unstable.

Let us now give a simple argument suggesting nonlinear instability of the shifted states (3.4) for $a<0$ under the NLS flow. If $K=1$, it follows from the momentum balance equation $(6.2)$ in Lemma 6.1 that the momentum $P(\Psi)$ defined by 6.1 is an increasing function of time if $m_{1}=0$ and a decreasing function of time if $m_{1}=1$, for the two choices in (3.6). Indeed, we obtain the following chain of transformations by using the boundary conditions in (2.2) and the constraint 2.10):

$$
\begin{aligned}
\frac{d}{d t} P(\Psi) & =(-1)^{m_{1}+1}\left[\sum_{j=2}^{N}\left|\psi_{j}^{\prime}(0)\right|^{2}-\sum_{j=2}^{N} \sum_{i=2}^{N} \frac{\alpha_{1}^{2 / p}}{\alpha_{j}^{1 / p} \alpha_{i}^{1 / p}} \psi_{j}^{\prime}(0) \bar{\psi}_{i}^{\prime}(0)\right] \\
& =(-1)^{m_{1}+1}\left(\sum_{j=2}^{N} \sum_{\substack{i=2 \\
i \neq j}}^{N} \frac{\alpha_{1}^{2 / p}}{\alpha_{i}^{2 / p}}\left|\psi_{j}^{\prime}(0)\right|^{2}-\sum_{j=2}^{N} \sum_{\substack{i=2 \\
i \neq j}}^{N} \frac{\alpha_{1}^{2 / p}}{\alpha_{j}^{1 / p} \alpha_{i}^{1 / p}} \psi_{j}^{\prime}(0){\overline{\psi_{i}}}_{i}^{\prime}(0)\right) \\
& =\frac{1}{2}(-1)^{m_{1}+1} \sum_{j=2}^{N} \sum_{\substack{i=2 \\
i \neq j}}^{N} \frac{\alpha_{1}^{2 / p}}{\alpha_{j}^{2 / p} \alpha_{i}^{2 / p}}\left|\alpha_{j}^{1 / p} \psi_{j}^{\prime}(0)-\alpha_{i}^{1 / p} \psi_{i}^{\prime}(0)\right|^{2}
\end{aligned}
$$

Hence $\frac{d}{d t} P(\Psi) \geq 0$ if $m_{1}=1$ and $\frac{d}{d t} P(\Psi) \leq 0$ if $m_{1}=0$.

Since the shifted states (3.4) satisfies $P(\Phi)=0$, monotonicity of the momentum $P(\Psi)$ in time $t$ immediately implies nonlinear instability of the shifted states (3.4) with $a<0$ under the NLS flow, despite that these shifted states are spectrally stable by Corollary 4.3. Indeed, if $a<0$ (or $\left.m_{1}=1\right)$, the value of the momentum $P(\Psi)$ is monotonically increasing in time as soon as the right-hand side of (7.4) is nonzero. Therefore, if $P(\Psi)$ is initially near zero, which is the value of $P(\Phi)$ for every shifted state $(3.4)$ with $a \in \mathbb{R}$, then $P(\Psi)$ grows far away from the zero value. This simple argument leads us to the following conjecture.

Conjecture 7.1. In the case $K=1$, the branch of shifted states (3.4) with a 0 is nonlinearly unstable under the NLS flow. 
We also add more details on the travelling waves of the homogeneous NLS equation on the infinite line given by 6.5 . Every stationary solution $U_{\text {stat }}(x) e^{i t}$ to the NLS equation (6.5) is translated into a family of the travelling solutions with speed $c$ by the Lorentz transformation

$$
U_{\text {trav }}(t, x)=U_{\text {stat }}(x-c t) e^{i t+i c x / 2-i c^{2} t / 4}, \quad x \in \mathbb{R}, \quad t \in \mathbb{R} .
$$

Computing the momentum $P(\Psi)$ given by (6.1) at the solution $\Psi$, which is defined by (6.8) with (7.5), yields

$$
P(\Psi)=\frac{c}{2} \alpha_{1}^{2 / p}(-1)^{m_{1}+1}\left\|U_{\text {stat }}\right\|_{L^{2}(\mathbb{R})}^{2}
$$

If $a<0$ (or $m_{1}=1$ ), then $\frac{d}{d t} P(\Psi) \geq 0$ and the increase of the momentum can be compensated by a travelling wave (7.5) moving with the speed $c>0$ as follows from (7.6). The maximum of the shifted state (3.4) with $a<0$ is located in the only incoming edge but it may move towards the vertex point at $x=0$ along the travelling wave (7.5).

On the other hand, if $a>0$ (or $m_{1}=0$ ), then $\frac{d}{d t} P(\Psi) \leq 0$ and the decrease of the momentum can be compensated by the same travelling wave (7.5) still moving with the speed $c>0$, as follows from (7.6). Therefore, we may anticipate that the maximum of the shifted state (3.4) first moves towards the vertex point at $x=0$ along the only incoming edge, then splits into $N-1$ maxima in the $N-1$ outgoing edges and these $N-1$ maxima keep moving outward the vertex point at $x=0$, subject to additional spectral instability due to symmetry-breaking perturbations between $N-1$ outgoing edges, according to Corollary 4.3. This dynamical picture is in agreement with the variational consideration above, but the proof of validity of this dynamical picture is beyond the scope of this work and will be considered elsewhere. Therefore, we summarize this dynamical picture as another conjecture.

Conjecture 7.2. In the case $K=1$, the shifted state (3.4) with a $<0$ leads to a solitary wave that moves towards the vertex point at $x=0$ along the only incoming edge, splits into $N-1$ solitary waves in the $N-1$ outgoing edges, which transform due to their spectral instability while moving outward the vertex point at $x=0$.

\section{REFERENCES}

[1] R. Adami, C. Cacciapuoti, D. Finco, and D. Noja, "On the structure of critical energy levels for the cubic focusing NLS on star graphs", J. Phys. A.: Math. Theor. 45 (2012), 192001 (7 pages).

[2] R. Adami, C. Cacciapuoti, D. Finco, and D. Noja, "Variational properties and orbital stability of standing waves for NLS equation on a star graph", J. Diff. Eqs. 257 (2014), 3738-3777.

[3] R. Adami, E. Serra, and P. Tilli, "Threshold phenomena and existence results for NLS ground states on graphs", J. Funct. Anal. 271 (2016), 201-223.

[4] G. Beck, S. Imperiale, and P. Joly, "Mathematical modelling of multi conductor cables", Discrete Contin. Dyn. Syst. Ser. S 8 (2015), 521-546.

[5] G. Berkolaiko and P. Kuchment, Introduction to quantum graphs, Mathematical Surveys and Monographs 186 (Providence, RI: AMS, 2013).

[6] G. Caputo and D. Dutykh "Nonlinear waves in networks: Model reduction for the sine-Gordon equation", Phys. Rev. E 90 (2014), 022912 (8 pages).

[7] T. Cazenave, Semilinear Schrödinger equations, Courant Lecture Notes in Mathematics 10 (New York University, Courant Institute of Mathematical Sciences, New York; AMS, Providence, RI, 2003).

[8] S. Cuccagna, D. Pelinovsky, and V. Vougalter, "Spectra of positive and negative energies in the linearized NLS problem", Comm. Pure Appl. Math. 58 (2005), 1-29.

[9] G.F. Dell'Antonio and E. Costa, "Effective Schrödinger dynamics on $\epsilon$-thin Dirichlet waveguides via quantum graphs: Star-shaped graphs”, J. Phys. A: Math. Theor. 43 (2010), 474014 (23 pages). 
[10] P. Exner and H. Kovarik, Quantum waveguides (Springer, Cham-Heidelberg-New York-Dordrecht-London, 2015).

[11] P. Exner and O. Post, "Approximation of quantum graph vertex couplings by scaled Schrödinger operators on thin branched manifolds", J. Phys. A: Math. Theor. 42 (2009), 415305 (22 pages).

[12] P. Exner and O. Post, "A general approximation of quantum graph vertex couplings by scaled Schrödinger operators on thin branched manifolds", Comm. Math. Phys. 322 (2013), 207-227.

[13] M. Grillakis, "Linearized instability for nonlinear Schrödinger and Klein-Gordon equations", Comm. Pure Appl. Math. 41 (1988), 747-774.

[14] M. Grillakis, J. Shatah, and W. Strauss, "Stability theory of solitary waves in the presence of symmetry", J. Funct. Anal. 74 (1987), 160-197.

[15] P. Joly and A. Semin, "Construction and analysis of improved Kirchoff conditions for acoustic wave propagation in a junction of thin slots" Paris-Sud Working Group on Modelling and Scientific Computing 2007-2008, pp. 4467 (ESAIM Proc. 25, EDP Sci., Les Ulis, 2008).

[16] P. Joly and A. Semin, "Mathematical and numerical modeling of wave propagation in fractal trees", C.R. Math. Acad. Sci. Paris 349 (2011), 1047-1051.

[17] A. Kairzhan, D. Pelinovsky, "Nonlinear instability of half-solitons on star graphs", arXiv:1706.00060 (2017)

[18] S. Kosugi, "Semilinear elliptic equations on thin network-shaped domains with variable thickness", J. Diff. Eqs. 183 (2002), 165-188.

[19] P. Kuchment, "Graph models for waves in thin structures, Waves Random Media" 12 (2002) R1-R24.

[20] S. Molchanov and B. Vainberg, "Scattering solutions in networks of thin fibers: small diameter asymptotics", Comm. Math. Phys. 273 (2007), 533-559.

[21] D.Noja, Nonlinear Schrödinger equation on graphs: recent results and open problems, Phil. Trans. R. Soc. A, 372 (2014), 20130002 (20 pages).

[22] J.A. Pava and N. Goloshchapova, "Extension theory approach in stability of standing waves for NLS equation with point interactions", arXiv:1507.02313 v2 (2017).

[23] O. Post, "Spectral convergence of quasi-one-dimensional spaces", Ann. Henri Poincaré 7 (2006), 933-973.

[24] K.K. Sabirov, D.B. Babajanov, D.U. Matrasulov, and P.G. Kevrekidis, "Dynamics of Dirac solitons in networks", arXiv:1701.05707(2017)

[25] Z. Sobirov, D. Babajanov, and D. Matrasulov, "Nonlinear standing waves on planar branched systems: Shrinking into metric graph", Nanosystems: Physics, Chemistry, Mathematics 8 (2017), 29-37.

[26] Z. Sobirov, D. Babajanov, D. Matrasulov, K. Nakamura, and H. Uecker, "Sine-Gordon solitons in networks: Scattering and transmission at vertices", EPL (Europhys. Lett.) 115 (2016), 50002 (6 pages).

[27] Z. Sobirov, D. Matrasulov, K. Sabirov, S. Sawada, and K. Nakamura, "Integrable nonlinear Schrödinger equation on simple networks: Connection formula at vertices", Phys. Rev. E 81 (2010), 066602 (10 pages)

[28] G. Teschl, Ordinary Differential Equations and Dynamical Systems, Graduate Studies in Mathematics 140 (AMS, Providence, RI, 2012).

[29] H. Uecker, D. Grieser, Z. Sobirov, D. Babajanov, and D. Matrasulov, "Soliton transport in tabular networks: transmission at vertices in the shrinking limit", Phys. Rev. E 91 (2015), 023209 (8 pages).

Department of Mathematics, McMaster University, Hamilton, Ontario L8S 4K1, Canada

E-mail address: kairzhaa@math.mcmaster.ca

Department of Mathematics, McMaster University, Hamilton, Ontario L8S 4K1, Canada

E-mail address: dmpeli@math.mcmaster.ca 